\title{
Advances in Liver Fibrosis Imaging and Hepatocellular Carcinoma: Update in 2013
}

\author{
Masatoshi Kudo \\ Kinki University School of Medicine, Osakasayama, Japan
}

The 3rd Asia-Pacific Primary Liver Cancer Expert Meeting (APPLE) was held on July 6-8, 2012, in Shanghai, China. In this meeting more than 50 invited international speakers and more than 500 participants from Asian countries participated. Highly scientific lectures followed by active discussion made this meeting an invaluable one.

The 48th Annual Meeting of the Liver Cancer Study Group of Japan was held on July 20-21, 2012, in Kanazawa, Japan. This meeting was also active and outstanding. This supplement issue contains selected articles with a high scientific value from these 2 meetings.

Fujimoto et al. [1] and Yada et al. [2] describe the usefulness of noninvasive techniques in evaluating liver fibrosis using real-time tissue elastography (RTE). RTE is a strain elastography in contrast to shear wave elastography in the diagnosis of liver stiffness. According to these 2 articles, RTE seems to be a promising method in evaluating liver fibrosis stage.

Kudo et al. [3] describe the role of gadolinum-ethoxybenzyl-diethylenetriamine imaging (Gd-EOB-DTPA MRI) in the management of hepatocellular carcinoma (HCC). This is a report based on the votes cast by 144 HCC experts at the 48th Annual Meeting of the Liver Cancer Study Group of Japan. The voting results which achieved more than $67 \%$ agreement are summarized as 'consensus statements'. Furthermore, issues which obtained more than $50 \%$ agreement are summarized as 'informative statements'. This report presents very interesting results and reflects daily clinical practice on use of EOB-MRI in Japan.

\section{KARGER}

Fax +4161306 1234

E-Mail karger@karger.com

www.karger.com
(C) 2013 S. Karger AG, Basel

0030-2414/13/0847-0001\$38.00/0

Accessible online at:

www.karger.com/ocl
Takayasu [4] contributes a state-of-the-art article on transcatheter arterial chemoembolization (TACE) for unresectable HCC. The author concluded that conventional TACE as well as drug-eluting beads loaded with doxorubicin and yttrium-90 $\left({ }^{90} \mathrm{Y}\right)$ microspheres demonstrated a similar median survival.

Kim and Kim [5] report that ${ }^{90} \mathrm{Y}$ radioembolization has a potent anticancer effect with negligible adverse events if appropriate pretreatment evaluations, including dosimetry, calculation of lung shunt fraction and assessment of vascular anatomy, are performed. They concluded that selected populations, for whom TACE would not be effective, are candidates for ${ }^{90} \mathrm{Y}$ radioembolization.

Peng and Chen [6] report that combination therapy of TACE with radiofrequency ablation (RFA) showed better survival and recurrence-free survival than an RFA alone group. Thus, they expect the combination therapy of TACE with RFA to become a standard of care for HCC, which is treatable with RFA, in the future.

Makino et al. [7] discuss how RFA can be performed for HCCs, which have poor conspicuity on grayscale ultrasonography (US), by introducing US fusion imaging with CT/MRI.

Inoue et al. [8] show that contrast-enhanced US (CEUS) can be used to assess the efficacy of RFA for HCC, with the potential of reducing the number of CT scans required for evaluating treatment response.

Minami and Kudo [9] examine therapeutic response assessment of TACE for HCC by US, CT and MRI. The authors conclude that CT is commonly used as the standard imaging technique, but Lipiodol makes it difficult to detect

Masatoshi Kudo, MD, PhD

Division of Gastroenterology and Hepatology, Department of Internal Medicine Kinki University Faculty of Medicine 377-2 Ohno-Higashi, Osakasayama, Osaka 589-8511 (Japan)

E-Mailm-kudo@med.kindai.ac.jp 
the residual tumor on CT. CEUS is a useful tool for assessing the vascularity of the viable tumor in comparison with CT. Dynamic MRI is also useful in demonstrating viable tumor with high sensitivity, but low specificity. We should keep in mind that these 3 techniques have both advantages and disadvantages in response evaluation after TACE.

Tsai et al. [10] report that serum/plasma markers, functional MRI and FDG-PET have been selectively used to predict disease outcome after radiotherapy to HCC.

Wei and Zeng [11] propose that external beam radiotherapy (EBRT) be included in the National Comprehensive Cancer Network since EBRT was more effective than sorafenib for improving patient survival when tested on tumors of comparable metastatic size.

Minata et al. [12] report that evaluating vascular endothelial growth factor in HCC tissue after surgical resection has predictive value for metastatic HCC recurrence. The ability to risk stratify should improve treatment strategies after hepatectomy.

Nishida et al. [13] discuss how methylation status of $A P C$ sequences could be a promising marker for improving HCC management when considering the strong association between the ratio of the methylated to unmethylated $A P C$ sequences in serum and the presence of portal vein thrombosis.
Minata et al. [14] show that expression levels of E-cadherin in adjacent noncancerous liver after surgical resection is associated with later metastatic HCC recurrence. Analysis of E-cadherin expression should provide important information for predicting recurrence after curative resection of HCC.

Nishida and Kudo [15] report that recent whole-genome analyses and exome sequencing of tumor DNA have revealed numerous novel alterations to cancer-related genes and pathways critical for HCC development. In addition, various risk factors for HCC, such as the presence or absence of hepatitis $B$ and hepatitis $C$ virus, may affect the mutation profile of the corresponding cancer genome. On the other hand, genome-wide association studies have also identified important single-nucleotide polymorphisms involved in HCC development, which may allow detection of a group at high risk of HCC emergence. Such analyses will clarify how this malignancy can be treated, diagnosed and prevented more effectively.

I believe this supplement issue contains articles with high scientific value and thus will prove to be beneficial for readers of Oncology.

\section{Disclosure Statement}

The author declares that no conflict of interests exist.

\section{References}

-1 Fujimoto K, Kato M, Kudo M, Yada N, Shiina $\mathrm{T}$, Ueshima $\mathrm{K}$, et al: Novel image analysis method using ultrasound elastography for noninvasive evaluation of hepatic fibrosis in patients with chronic hepatitis C. Oncology 2013;84(suppl 1):3-13.

2 Yada N, Morikawa H, Fujimoto K, Kato M, Kawada N, Kudo M: Assessment of liver fibrosis with real-time tissue elastography in chronic viral hepatitis. Oncology 2013;84 (suppl 1):14-20.

$\checkmark 3$ Kudo M, Matsui O, Sakamoto M, Kitao A, Kim T, Ariizumi S, et al: Role of gadoliniumethoxybenzyl-diethylenetriamine pentaacetic acid-enhanced magnetic resonance imaging in the management of hepatocellular carcinoma: consensus at the symposium of the 48th Annual Meeting of the Liver Cancer Study Group of Japan. Oncology 2013;84 (suppl 1):21-27.

4 Takayasu K: Transcatheter arterial chemoembolization for unresectable hepatocellular carcinoma: recent progression and perspective. Oncology 2013;84(suppl 1):28-33.

$>5$ Kim YH, Kim DY: Yttrium-90 radioembolization for hepatocellular carcinoma: what we know and what we need to know. Oncology 2013;84(suppl 1):34-39.
-6 Peng ZW, Chen MS: Transcatheter arterial chemoembolization combined with radiofrequency ablation for the treatment of hepatocellular carcinoma. Oncology 2013;84 (suppl 1):40-43.

$\checkmark 7$ Makino Y, Imai Y, Ohama H, Igura T, Kogita S, Sawai Y, et al: Ultrasonography fusion imaging system increases the chance of radiofrequency ablation for hepatocellular carcinoma with poor conspicuity on conventional ultrasonography. Oncology 2013;84(suppl 1):44-50.

$>8$ Inoue T, Kudo M, Hatanaka K, Arizumi T, Takita M, Kitai S, et al: Usefulness of contrast-enhanced ultrasonography to evaluate the post-treatment responses of radiofrequency ablation for hepatocellular carcinoma: comparison with dynamic CT. Oncology 2013;84(suppl 1):51-57.

-9 Minami Y, Kudo M: Therapeutic response assessment of transcatheter arterial chemoembolization for hepatocellular carcinoma: ultrasonography, CT and MR imaging. Oncology 2013;84(suppl 1):58-63.

10 Tsai CL, Koong AC, Hsu FM, Graber M, Chen IS, Cheng JCH: Biomarker studies on radiotherapy to hepatocellular carcinoma. Oncology 2013;84(suppl 1):64-68.
11 Wei J, Zeng ZC: Is it time to adopt external beam radiotherapy in the NCCN guidelines as a therapeutic strategy for intermediate/ advanced hepatocellular carcinoma? Oncology 2013;84(suppl 1):69-74.

12 Minata M, Harada KH, Kudo M, Ikai I, Nishida N: The prognostic value of vascular endothelial growth factor in hepatocellular carcinoma for predicting metastasis after curative resection. Oncology 2013;84(suppl 1):75-81

13 Nishida N, Arizumi T, Takita M, Nagai T, Kitai S, Yada N, et al: Quantification of tumor DNA in serum and vascular invasion of human hepatocellular carcinoma. Oncology 2013;84(suppl 1):82-87.

14 Minata M, Kudo M, Harada KH, Ikai I, Nishida N: Expression of E-cadherin and vascular endothelial growth factor in noncancerous liver is associated with recurrence of hepatocellular carcinoma after curative resection. Oncology 2013;84(suppl 1):86-90.

15 Nishida N, Kudo M: Recent advancements in comprehensive genetic analyses for human hepatocellular carcinoma. Oncology 2013; 84(suppl 1):91-95. 